\title{
Criminologie
}

\section{Réflexion autour de la notion de services aux femmes dans le système de justice}

\section{Renée Collette-Carrière}

Volume 16, numéro 2, 1983

Les femmes et la justice pénale

URI : https://id.erudit.org/iderudit/017184ar

DOI : https://doi.org/10.7202/017184ar

Aller au sommaire du numéro

Éditeur(s)

Les Presses de l'Université de Montréal

ISSN

0316-0041 (imprimé)

1492-1367 (numérique)

Découvrir la revue

Citer cet article

Collette-Carrière, R. (1983). Réflexion autour de la notion de services aux femmes dans le système de justice. Criminologie, 16(2), 101-111.

https://doi.org/10.7202/017184ar d'utilisation que vous pouvez consulter en ligne.

https://apropos.erudit.org/fr/usagers/politique-dutilisation/ 
RÉFLEXION AUTOUR DE LAA NOTION DE SERVICES AUX FEMMES DANS LE SYSTĖME DE JUSTICE Renée Collette-Carrière*

Femmes! Services! Justice! Trois mots! Trois réalités! Quels liens entre ces réalités? Quels rapports peut-on établir entre ces mots? Peut-on les associer sans jeu de mots? Sont-ils conciliables? et de quoi parlons-nous au juste? Des femmes et de leurs rapports avec le système de justice. De leurs rapports avec les ressources communautaires ou non, les services générés par ce système.

Prenons pour acquis que les femmes puissent avoir des démêlés avec la justice. Qu'arrive-t-il? Comment, dans et autour de ce système, se sont organisés et s'articulent les services par rapport à la clientèle féminine? Comment donc s'est développée la notion de service, la notion de service aux femmes et surtout à celles qui sont dans le système de justice? C'est à travers une analyse historique de la condition des femmes et de l'assistance sociale que nous tenterons de cerner la portée des questionnements que nous avons énoncés et les implications qu'ils suscitent pour l'avenir.

\section{DE LA FRANCE À LA TERRE D'AMÉRIQUE}

La Nouvelle-France, terre de déploiement de ressources insoupçonnées, d'initiatives imprévues dans la mère-patrie, cette Neuve-France, a accueilli des femmes actives, innovatrices et autonomes. Les modes de vie inhérents à l'établissement du pays neuf, s'articulaient selon un modèle impensable pour l'époque, du moins en ce qui concerne la vie des femmes. Aux $17^{\circ}$ et $18^{\circ}$ siècles, la condition féminine y est toujours différente de celle vécue en France. Les femmes qui ont quitté le continent européen l'ont fait, selon les historiennes du Collectif Clio (1982), soit pour accompagner un époux, en trouver un ou en vue d'actions différentes telles la fondation d'œuvres sociales ou d'éducation. Pour affronter périple si dur, elles étaient sûrement des personnes déterminées, autonomes et indépendantes. Elles ont eu à faire face à une vie rude, semée d'embûches. Leur rôle a été plus large que celui dévolu traditionnellement aux femmes. Il est à souligner que dans toute nouvelle civilisation ou

* Chargée de cour à l'École de criminologie et à la faculté d'éducation permanente, Université de Montréal. 
organisation, semblable constatation peut être faite. Ainsi, leur sphère d'activités est étendue. Les tâches ne sont pas divisées selon le sexe. Il y a partage. Cela ne signifie pas que les hommes effectuent les tâches traditionnellement féminines (ménage, cuisine, couture). Cela implique plutôt que les femmes accomplissent des tâches d'hommes (défricher, brûler, piocher, construire, récolter, écorcher des peaux, calfeutrer). Bâtir un pays demande toutes les énergies possibles. Cette conjoncture a permis que les femmes ne soient plus limitées au domaine du privé mais qu'elles agissent dans la sphère du public sans pour autant remettre en question la conception de l'inégalité des sexes. Responsables de la communauté en l'absence des hommes partis guerroyer, trapper ou en voyage en France, elles ont eu des droits comme celui de voter, des pouvoirs que le $19^{\mathrm{e}}$ siècle viendra leur ôter et qu'elles n'avaient pas dans leur pays d'origine.

Avec la colonisation, un courant religieux important était présent. Parmi les arrivant(e)s, bon nombre venaient évangéliser les peuplades païennes. À ce chapitre, des femmes ont été très actives. Bien sûr, le mariage constituait la voie la plus logique et la plus probable pour une fille. Cependant, celle qui ne se mariait pas volontairement ou non, pouvait trouver dans la vie religieuse une façon d'œuvrer fort utilement dans une société en quête de tout. La Nouvelle-France a ainsi vu de ses filles devenir d'importantes fondatrices et dirigeantes d'œuvres d'éducation, de santé et d'action sociale auprès des démuni(e)s. Ces religieuses «ont joué un rôle $d^{\prime}$ une importance sociale indiscutable... Dès la fin du $17^{\mathrm{e}}$ siècle, ce sont des communautés de femmes qui ont assumé, en NouvelleFrance, tout ce qui concernait la charité publique : secours aux pauvres, vieillards, invalides, malades, fous, prisonnières, prostituées, orphelins». (Le Collectif Clio, 1982, p. 47). Ces femmes ont continué à travailler, à innover et à mobiliser d'autres femmes au cours des siècles qui ont suivi.

\section{SOCIÉTÉ NOUVELLE, SERVICES À BÂTIR}

C'est dans ce contexte que se sont organisés les services dans plusieurs domaines dont celui des problèmes sociaux et de la justice. Afin d'analyser plus longuement le rôle des femmes et de l'organisation des services pour elles, jetons un bref regard sur le développement de l'assistance sociale comme telle.

À mesure que l'organisation sociale s'élabore, certains problèmes surgissent. Ils touchent plus durement certains groupes et les femmes n'y échappent pas (Le Collectif Clio, 1982). Comment cette 
société nouvelle réagit-elle? Sous le Régime français, l'assistance sociale apparaît. Elle a d'abord et «longtemps découlé du désir d'indépendance et de solidarité interne de la famille, en même temps que de l'inspiration active du clergé» (Rapport du Comité d'étude sur l'Assistance publique, 1963, p. 27). C'est donc dans un climat de solidarité et d'hospitalité très fort que s'organise cette aide. Elle est essentiellement privée et locale. Les membres de la communauté y contribuent largement et les congrégations religieuses y sont très actives. Après la conquête, il n'y a pas au Québec de changement immédiat. «Au contraire, le climat de crainte et la peur d'une ingérence indue de la part des nouveaux gouvernants ont contribué à renforcer les liens communautaires et à rendre davantage privées et personnelles les formes de secours» (ibid., p. 29). Vers la fin du $18^{\mathrm{e}}$ siècle, l'État donne quelques subsides à des œuvres hospitalières pour qu'elles s'occupent des malades mentaux et des enfants abandonnés. Se dessine donc un peu une participation gouvernementale. C'est l'Acte de l'Amérique du Nord britannique qui édictera la responsabilité provinciale en matière de bien-être et de santé (art. 92). Les municipalités s'impliquent aussi dans ce domaine à partir du $19^{\mathrm{e}}$ siècle.

La première loi québécoise de l'assistance publique a été promulguée en 1921. Selon les analystes, elle était devenue «une nécessité historique en même temps qu'une tentative de systématisation de l'assistance gouvernementale aux institutions de bienfaisance». (Rapport du Comité d'étude sur l'assistance publique, 1963, p. 32). Ces dernières demeuraient privées cependant. Cette loi sera en vigueur jusqu'en 1960. Elle aura subi quelques modifications au cours des années sans en transformer la portée.

La fin des années cinquante et le début des années soixante verront des changements importants. Ceux-ci auront comme résultat de rendre publics les domaines de l'assistance sociale, de l'éducation et de la santé et d'y apporter des réformes notoires. Le rôle supplétif qu'assumait l'État jusqu'alors sera dévolu au secteur privé comme les organismes de charité. C'est la Révolution tranquille. Il y a une évolution globale. En juin 1963, le Comité d'étude sur l'assistance publique dépose son rapport. Ses recommandations renforcent les changements amorcés. Le secteur privé continue d'exister mais dans certaines limites. Ultérieurement, ces transformations seront complétées avec les recommandations de la Commission d'enquête sur la santé et le bien-être social (Castonguay-Neveu) créée en 1966. Elle propose à la fin des années soixante-dix, «un nouveau modèle» de 
services sociaux (Renaud, 1978, p. 140) et une mainmise encore plus grande de l'État.

Nous sommes donc dans une situation où l'État joue un rôle prépondérant en termes de définisseur et de dispensateur de services. Comment cette situation a-t-elle évolué du côté des femmes compte tenu du contexte? Plus particulièrement, comment s'articule-t-elle dans le système de justice?

\section{LE DÉVELOPPEMENT DES SERVICES AUX FEMMES}

Nous avons très brièvement décrit la condition féminine des premiers temps du Régime français et combien originale a été la contribution des femmes. Nous savons qu'elles ont été fondatrices et dirigeantes d'œuvres qui ont connu dans les siècles à venir, un développement fort important et essentiel tant dans le domaine de la santé, de l'éducation que du service social ou de l'assistance sociale. Relevant de la charité publique et laissée à l'initiative privée, cette assistance, ainsi que nous venons brièvement d'en prendre connaissance, s'est développée au sein des communautés locales.

Dans le secteur de la justice, l'aide à ceux et celles qui ont commis un crime se confond dans les premiers temps de ce pays neuf comme parfois aujourd'hui, avec celle fournie aux pauvres, aux malades, aux personnes abandonnées, etc.

L'implication féminine à ce niveau est très importante. Les problèmes de pauvreté amènent beaucoup de détresse pour les femmes. Au $19^{e}$ siècle par exemple, la situation est particulièrement pénible et les religieuses ne peuvent suffire à la tâche. Des femmes de la bourgeoisie fondent certains organismes bénévoles principalement au début du siècle du côté des anglophones : Female Benevolent Society (1817), Female Compassionate Society (1822) à Québec et le Montreal Ladies Benevolent Society (1824). Les religieuses s'impliquant de plus en plus auprès des femmes ayant des démêlés avec la justice. Elles visitent les asiles ou prisons qui, dans ces temps lointains abritaient tant «les femmes de mauvaise vie ou furieuses» que les indigents et les criminels des deux sexes (Berzins et ColletteCarrière, 1979, p. 88). Elles viennent en aide à celles qui sortent de prison en fondant des refuges, sortes d'ancêtres à nos actuelles maisons de transition. Puis elles font des pressions tant auprès des autorités ecclésiastiques que gouvernementales pour construire une prison féminine qui ouvre ses portes en septembre 1876 . Les religieuses du Bon Pasteur pouvaient ainsi continuer à «travailler au relèvement moral des prisonnières» (Sr Marie-de-Saint-Benoît, 1953, p. 8) dans 
cette institution appelée Asile Ste-Darie, prison des femmes. Connue aussi sous le nom de Prison des femmes catholiques à Montréal, cette prison provinciale était communément nommée «Fullum». Cette œuvre avait reçu l'aide gouvernementale mais, en 1891, les religieuses en devinrent les seules propriétaires et directrices. Elles purent ainsi varier leurs actions en opérant une buanderie commerciale qui donnait du travail à certaines détenues, en construisant la Maison Ste-Hélène pour les ex-détenues, devenue plus tard une institution pour jeunes délinquantes. Les objectifs visés s'articulaient autour des valeurs attachées au rôle féminin traditionnel et de programmes conformes à cette conception. Jusqu'en 1960, cette communauté religieuse féminine s'occupa ainsi des femmes détenues et ex-détenues.

En 1960, le gouvernement en reprit la direction dans le cadre des réformes dont nous avons fait mention précédemment. Une nouvelle prison fut bâtie, La Maison Tanguay, qui ouvrit ses portes en 1964.

Nous avons dit que les communautés religieuses étaient souvent secondées de bénévoles laïques. Ce travail bénévole a été et est encore la fonction de beaucoup de femmes. Certaines agences sociales privées ont été aussi fort actives dans le domaine de l'aide aux femmes aux prises avec la justice, par exemple la Société d'Orientation et de Réhabilitation sociale (S.O.R.S.), John Howard Society, Elizabeth Fry Society Assistance aux femmes à la cour, prise en charge, visites de libérées conditionnelles, etc. Leur implication s'est poursuivie au-delà des années 60 .

Nous pouvons donc constater le rôle important des femmes dans le domaine de la justice. Rôle récupéré dans une large mesure par l'État dans la vague des réformes de la Révolution tranquille. Cette récupération n'a cependant pas signifié une amélioration des services dans le système de justice en ce qui concerne les femmes mais a amené une approche différente, plus bureaucratique, moins adaptée.

\section{SYSTÈME DE JUSTICE, SERVICES CORRECTIONNELS ET FEMMES}

Les services correctionnels conviennent-ils aux femmes? Nos interventions auprès des femmes ayant des démêlés avec la justice sont-elles sources de mieux-être, de réinsertion sociale?

Nous répondons négativement à ces questions. La mise en place de services dans le domaine de la justice en général et dans celui 
des services correctionnels a été élaborée à partir d'une conception patriarcale. Ces services ne sont qu'un calque d'un système pensé et conçu par et pour les hommes. Même notre apprentissage en tant qu'intervenants va dans ce sens. Ainsi, les modèles que l'on essaie de reproduire ne satisfont ni la femme-intervenante, ni la femmecliente non plus que l'homme-intervenant face à la femme-cliente. Ce dernier se sent tout autant dépourvu. Il faut ici cesser de séparer ce que nous sommes de ce que nous savons ${ }^{1}$.

On n'a pas été à l'écoute de la parole des femmes et de leurs besoins. Parole non dite la plupart du temps! Besoins non pas seulement méconnus mais non ressentis. Il faut apprendre à exprimer et à comprendre pour aider et agir. Dans le domaine de la justice, il y a distance entre la réalité et les besoins. Les attitudes paternalistes largement mises en évidence par plusieurs études tant au Canada (Bertrand, 1979), aux États-Unis (Feinman, 1980), en Angleterre (Smart, 1976) qu'ailleurs ${ }^{2}$ expliquent jusqu'à un certain point cet écrit. On finit par avoir un système discriminatoire en ce sens qu'il n'offre pas aux femmes en termes de services, les mêmes possibilités qui existent du côté masculin. On a des difficultés à faire admettre aux différents paliers décisionnels, la nécessité même de ces services et de leurs développements.

Illustrons brièvement nos propos. Le Service correctionnel fédéral, domaine souvent analysé et critiqué tant par des rapports de commissions d'enquête que par des analyses de l'extérieur au sujet de ses programmes of ferts aux détenues ${ }^{3}$, a fait la manchette en décembre 1981. Pourquoi? Parce que la Commission canadienne des droits de la personne, saisie d'une plainte du groupe «Women for Justice» déclarait qu'à la suite de son enquête, elle trouvait le Service correctionnel canadien coupable de discrimination à l'endroit des femmes détenues au pénitencier fédéral féminin de Kingston en Ontario (Berzins et Cooper, 1982). Seule institution fédérale du genre à desservir une population féminine, elle est donc inadéquate. Elle n'offre pas de services appropriés aux besoins de ces femmes

1. Adaptation d'une phrase de Christiane Olivier (1980) : «Je renonce à séparer ce que je suis de ce que je sais», p. 12.

2. Voir pour la France : Erhel et Leguay (1977) ; pour la Belgique : Rosart (1982) ; pour la Pologne : Plenska (1980) ; pour la Russie : Le Collectif l'Almanach (1980).

3. Pour de plus amples détails voir Berzins et Collette-Carrière (1979) et Berzins et Cooper (1982). 
détenues pour plus de deux ans et ceux qu'elle dispense, sont inférieurs en quantité (toute proportion gardée) et en qualité à ceux offerts aux hommes dans le même système, ce qui n'est pas peu dire. Bien sûr, les autorités ont reconnu avant même ce jugement qu'il y avait place pour des améliorations. Cependant, il n'y a jamais eu ni volonté politique ferme ni efforts sérieux pour agir. On a tergiversé trop longtemps sur la fermeture ou non de ce pénitencier, sur la prise en charge totale ou partielle par les gouvernements provinciaux de cette catégorie de détenues. On n'a pas pris les moyens concrets pour mettre un terme à cette situation anachronique et discriminatoire. Il faut ici une volonté politique. Le petit nombre de femmes, 200 sur 10000 détenus (août 1981) ne doit pas justifier l'inertie mais être plutôt source de solutions originales. Comme l'affirmait Feinman (1980), les changements dans les prisons féminines depuis 100 ans sont réels mais ils sont insuffisants et lents et les prémisses de base persistent toujours. Berzins et Cooper (1982) quant à elles souhaitent qu'en dénonçant les failles d'un tel système, on ne mette pas en place un système parallèle aussi sexiste mais qu'émerge plutôt le développement d'une approche humaniste de la planification dans le champ de la justice sociale.

Discrimination seulement au fédéral! Non, au provincial, il nous semble que les services offerts ne répondraient pas davantage aux besoins réels. La programmation est basée sur la même conception traditionnelle du rôle de la femme. Il s'agit de la reproduction de l'unjvers domestique et l'approche résulte en une infantilisation des femmes. Certains efforts sont faits mais on peut se demander si les objectifs visés sont les bons. Il y a une telle distance entre la réalité vécue comme détenue et les besoins, que nous pouvons nous questionner sur l'efficacité réelle d'un tel système. Il faut faire ce questionnement autrement qu'en termes de contrôle. Nous pouvons toujours nous persuader qu'en autant qu'il n'y a pas d'incident, de trouble, nous sommes efficaces. Mais avons-nous réellement atteint un objectif à plus long terme soit la réinsertion sociale de ces femmes détenues? Avons-nous contribué à leur fournir les outils adéquats pour qu'elles deviennent des citoyens autonomes et responsables? Avons-nous répondu à leurs besoins? Les connaissons-nous ces mêmes besoins?

Allons plus loin! La discrimination dont nous discutions précédemment aurait-elle un impact sur la récidive? Nous entendons souvent dire : "Ah! Ce sont toujours les mêmes femmes qui reviennent!» Aux États-Unis, Diane K. Lewis (1982) affirme que les hauts 
taux de récidive ( 50 à $85 \%$ ) des détenues américaines libérées des prisons, sont une conséquence des politiques discriminatoires et du peu d'implication des agences communautaires. Traditionnellement "ce syndrôme de portes tournantes est habituellement attribué aux caractéristiques personnelles de la détenue ou aux conditions de l'incarcération ${ }^{4} »$. Mais il y a plus. Cette explication est trop simple. Son étude porte sur les besoins réels, les types de services disponibles tant à la prison que dans la communauté et l'accessibilité réelle à ces services durant l'incarcération et lors de la libération. Ses résultats indiquent que le taux d'échec est souvent dû aux barrières que les détenues rencontrent soit la piètre qualité des ressources et l'indifférence générale face à leurs besoins. Il y a donc lieu de questionner fort sérieusement le système non seulement correctionnel mais tous les paliers du système judiciaire et des rapports qu'il entretient avec les fermmes.

\section{BARRIÈRES DRESSÉES CONTRE LE DÉVELOPPEMENT DES SERVICES}

Tentons maintenant de discuter de certains facteurs affectant la mise en place des services aux femmes et leur développement. Lewis (1982) en identifie plusieurs. Nous en avons ajouté d'autres découlant de nos expériences et lectures.

D'abord la question financière. Comment réunir les fonds nécessaires pour pouvoir offrir un service adéquat? Comment obtenir des budgets permettant de développer des services, des programmes à un palier quelconque du système de justice? Comment obtenir des fonds pour identifier les besoins et évaluer les ressources existantes? Jusqu'à quel point, les situations de crise économique et les politiques de restrictions budgétaires influencent-elles l'allocation des fonds. Et dans cette même veine, un autre facteur nous paraît très important, soit la définition des priorités et les critères d'éligibilité pour l'allocation des ressources financières.

Ensuite, la place que les organismes gouvernementaux accordent au privé. La connaissance des programmes gouvernementaux et privés distribuant des fonds. Des facteurs liés à la planification ellemême peuvent être identifiés. Celle-ci peut être inadéquate en raison des communications boiteuses entre la communauté et les clientes

4. Lewis (1982) p. 40. Traduction libre de : "This «revolving door syndrome» is usually attributed to the personal of female inmates or to the conditions of incarceration.» 
éventuelles de ces services, d'une mauvaise coordination ou même d'une certaine méfiance. Elle peut aussi à ce chapitre résulter d'un manque d'information auprès des utilisatrices (détenues, libérées conditionnelles) qui fait que ces dernières n'atteignent pas leurs objectifs, car elles ne connaissent pas les ressources pouvant les aider. Ceci s'applique aussi aux intervenants. Dans une autre optique, un désintérêt et une insensibilité de la part de la communauté peut jouer contre les bénéficiaires. Ainsi un certain ostracisme à l'endroit des femmes ayant des démêlés avec la justice peut leur être préjudiciable.

À cet égard, il convient de souligner la ténacité de la société Élizabeth Fry du Montréal Métropolitain. Traditionnellement, les sociétés Élisabeth Fry s'occupent du domaine des femmes et de la justice. La fondatrice, Elizabeth Fry, (1780-1845), s'avère une pionnière dans ce domaine. Témoin scandalisé des conditions de détention des femmes dans une prison britannique, elle contribue par son action concrète à leur donner espoir. À l'image des pionnières religieuses et laiques des premiers temps de la colonie, elle est sortie du rôle traditionnel de l'époque et a remis en cause la conception de l'emprisonnement pour les femmes.

La maison de transition Thérèse Casgrain constitue un exemple de cette volonté d'offrir aux femmes une ressource adéquate. Fondée par la Société Elizabeth Fry, cette maison est la seule au Québec qui accueille des femmes. Pendant des années, un groupe de personnes s'est battu pour qu'un service déjà offert aux hommes soit accessible aux femmes. Pourtant la reconnaissance de besoins spécifiques est encore fort peu admise; il y a place pour moultes actions mais le financement a été et est encore très difficile.

D'autres domaines peuvent aussi servir d'exemple. Ainsi du côté de la violence subie par les femmes, il a fallu que des femmes, très souvent bénévoles, initient des actions et mettent en place des services avec très peu de moyens.

Toutes ces ressources sont vulnérables à cause de la précarité des moyens financiers. Leur reconnaissance et leur survie sont pourtant essentielles à une amélioration des services auprès des femmes dans le domaine de la justice.

\section{ET L'AVENIR}

Il faut établir un consensus autour d'un point : le droit pour les femmes à des opportunités équivalentes dans le système de justice et donc à des services de qualité. Ces services ne doivent ni copier des 
modèles bâtis pour des populations masculines ni enfermer les femmes dans un ghetto idéologique mais s'insérer dans une société ayant pour principe la reconnaissance de l'égalité des hommes et des femmes.

Pour que ces services soient vraiment adéquats, il faut regarder ainsi que le font Berzins et Cooper (1982) les femmes aux prises avec la justice comme membres non pas de la population criminelle mais de la communauté des femmes. Leurs problèmes et leurs besoins, comme leur criminalité sont proches de leur condition de femmes et donc de leur appartenance à ce groupe. Des recherches doivent être initiées dans cette perspective. Il faut envisager les solutions dans ces termes et développer la solidarité des femmes et de l'ensemble de la communauté. Ainsi des services mis sur pied pour des femmes non criminelles pourraient être utiles sous certains rapports aux femmes criminelles et l'argument des coûts tombe. En parlant des coûts, Berzins et Cooper (1982) notent que les femmes constituent la moitié de la population et que «leur remarquable sous-représentation dans la population criminelle fait économiser aux payeurs de taxes des millions de dollars annuellement ${ }^{5}$ ". Certains programmes existants paraissent mal répondre à certains besoins identifiés, par exemple les problèmes de toxicomanie chez les femmes détenues. Pourquoi ne pas permettre le développement de services adéquats et ne pas reconnaître certains besoins spécifiques. D'aucuns affirmeront qu'il y a danger de discrimination à l'envers. Nous préférons parler d'une période transitoire, de rattrapage où une discrimination positive s'exercera. Elle est reconnue de plus en plus comme solution dans d'autres domaines et inscrite dans des textes légaux. C'est une question d'éthique!

Mais avant tout, établissons une démarche permettant d'identifier les besoins réels en laissant la parole à celles qui sont impliquées dans le domaine de la justice à titre d'utilisatrices ou d'intervenantes pour qu'ensemble elles puissent élaborer des services adéquats.

Il nous faudra du courage et de la ténacité. Refuser d'entendre cette parole des femmes en milieu de justice, en réduire la portée politique, ne l'écouter qu'avec compassion ou indifférence ne seraient qu'attitudes témoignant d'un sens restreint de la notion de justice.

5. "Their remarkable underrepresentation in the criminat poulation is saving the tax payers millions of dollars annually." 


\section{BIBLIOGRAPHIE}

BERTRAND, M.A. (1979), La femme et le crime, Montréal, L'Aurore.

BERZINS, L., R. COLLETTE-CARRIERE, (1979). «La femme en prison, un inconvénient social?", Santé mentale au Québec, 4, 2, p. 87-103.

BERZINS, L. Sh. COOPER (1982). «The political economy of correctional planning for women: The case of the bankrupt bureaucracy», Revue canadienne de criminologie, 24, 4, p. 399-416.

ERHEL, C., C. LEGUAY (1977). Prisonnières, Paris, Voix de femmes, Stock 2.

FEINMAN, C. (1980). Women in the criminal justice System, New York: Praeger.

LE COLLECTIF CLIO (1982). L'histoire des femmes au Québec depuis quatre siècles, Collection idéelles, Montréal, Quinze.

LE COLLECTIF L'AlMANACH (1980). Femmes et Russie 1980, Paris, Des Femmes.

LEWIS, D.K. (1982). «Female Exoffenders and Community Programs : Barriers to Service", Crime and delinquency, 28, 1, p. 40-51.

OLIVIER, C. (1980). Les enfants de Jocaste, Paris, Denoël/Gonthier.

PLENSKA, D. (1980). «La criminalité féminine en Pologne», Revue canadienne de criminologie, 22, 4, p. 464-475.

RAPPORT DU COMITÉ D'ÉTUDE SUR L'ASSISTANCE PUBLIQUE (1963). Gouvernement du Québec.

RENAUD, G. (1978). L'éclatement de la profession en service social, Montréal, Les Éditions coopératives Albert St-Martin.

ROSART, R. (1982). "Le monde des prisons. 11 - Seulement 217 femmes et 5 bébés", La libre Belgique, août.

SMART, C. (1976). Women, Crime and Criminology, a Feminist Critique, Londres, Routeldge et Kegan Paul.

SR. MARIE-DE-SAINT-BENOIT, r.b.p. (1953). Maison Ste-Darie ou La prison des femmes catholiques à Montréal, 1876-1952. Département de Service social, Faculté des arts et des sciences, Université de Montréal. Thèse inédite. 